\title{
Occurrence of mesangial IgA and IgM deposits in a control necropsy population
}

\author{
R SINNIAH́
}

From the Department of Pathology, National University of Singapore, Singapore General Hospital, Singapore 0316

SUMMARY Kidney sections were obtained from 200 consecutive control necropsies of patients who $\hat{\omega}$ died of traumatic injuries, with no clinical history of renal disease or other organic disease $\dot{\omega}$ discovered at necropsy. Mesangial IgA as the predominant immunoglobulin was found in $8 / 200 \mathrm{~N}$ $(4 \%)$ cases, with accompanying IgM in two of them; and IgM alone in two (1\%) subjects. Deposits $\%$ of $\mathrm{C} 3$ alone in blood vessels was observed in nine $(4.5 \%)$ cases. The glomerular morphology was $\frac{}{5}$ essentially normal or minor change only, with one case showing diffuse mesangial hypercellularity.

The high incidence of mesangial IgA deposits in the local apparently healthy population may reflect some common feature of the antigen(s) or complex involved. They may be of $\stackrel{\rho}{\sim}$ environmental, dietary or infectious origin. It is possible that many of these "spontaneous" $\vec{\bullet}$ deposits in the glomerular mesangium may result from the clearance of circulating nonnephritogenic immune complexes.

Mesangial IgA deposits have been reported as the commonest immunofluorescent finding in adult patients with idiopathic glomerular disease in France, ${ }^{1-4}$ Japan $^{5-7}$ and Singapore ${ }^{8}$ accounting for $33.7 \%$ of all local cases. It is found as a frequent glomerular immunoprotein deposit in asymptomatic microscopic haematuria, ${ }^{9}$ and recurrent proteinuria. $^{10}$

Investigation of the prevalence of mesangial IgA and other glomerular immunoprotein deposits in a normal live population is of course not possible. The present study was undertaken to determine the presence of immunoglobulin and complement deposits in the kidneys of a control necropsy population who died from traumatic injuries, with no clinically apparent symptoms of renal disease, and death was not due to organic disease. This group closely resembles an apparently healthy local population.

\section{Material and methods}

\section{POPULATION SELECTION}

Kidney sections were obtained at necropsy from 200 patients, 153 males and 47 females, for histological and immunofluorescent microscopic examination. All necropsies were carried out at the office of the

Accepted for publication 5 April 1982
Chief Forensic Pathologist of the Ministry of Health of Singapore between December 1978 and June 1981. Patients were selected consecutively who had no known history of renal or other disease, and deathso were due to accidents or suicide. Narcotic or drug overdose, alcoholism, and medical illness were excluded from the study. Past medical history, and. necropsies revealed no evidence of renal or othero organic disease, and macroscopically the kidneys:appeared normal. Portions of kidney were obtained within $24 \mathrm{~h}$ of death. The ages of the 200 consecutive, apparently disease-free cases ranged from 11 to $80 \mathrm{yr}, 3$ with a mean age of $35 \cdot 1 \mathrm{yr} \pm$ SD $16.6 \mathrm{yr}$. There wereo 146 Chinese, 22 Malays, 30 Indians and 2 Caucasians? reflecting the racial distribution of the Singaporeo population.

LIGHT MICROSCOPY
All renal specimens were fixed in Bouin's solution, స్ల embedded in paraffin and sectioned at $2 \mu \mathrm{m}$. The sections were stained with haematoxylin and eosin $\left(\mathrm{H}^{<}\right.$ and $E$ ), periodic acid-Schiff (PAS), periodic acide silver methenamine (PASM), Masson's trichromes? and Martius scarlet blue (MSB) stains.

\section{IMMUNOFLUORESCENCE MICROSCOPY}

Specimens of kidney were examined for direct伭 immunofluorescence by standard technique with specificity controls of the sera as described else- 
Data on control necropsy cases with mesangial IgA and IgM deposits

\begin{tabular}{|c|c|c|c|c|c|c|c|c|c|c|c|}
\hline \multirow{2}{*}{$\begin{array}{l}\text { Case } \\
\text { No }\end{array}$} & \multirow[t]{2}{*}{$\operatorname{Sex}$} & \multirow{2}{*}{$\begin{array}{c}\text { Age } \\
(y r)\end{array}$} & \multirow[t]{2}{*}{ Race } & \multirow[t]{2}{*}{ Cause of death } & \multicolumn{6}{|c|}{ Mesangial immunoprotein deposits } & \multirow[t]{2}{*}{ Glomerular morphology } \\
\hline & & & & & $\operatorname{Ig} A$ & IgG & IgM & C3 & $C l q$ & Fibrin & \\
\hline $\begin{array}{r}1 \\
2 \\
3 \\
4 \\
5 \\
6 \\
7 \\
8 \\
9 \\
10\end{array}$ & $\begin{array}{l}\mathbf{M} \\
\mathbf{M} \\
\mathbf{M} \\
\mathbf{F} \\
\mathbf{M} \\
\mathbf{M} \\
\mathbf{F} \\
\mathbf{F} \\
\mathbf{M} \\
\mathbf{F}\end{array}$ & $\begin{array}{l}26 \\
22 \\
31 \\
28 \\
30 \\
27 \\
22 \\
50 \\
25 \\
70\end{array}$ & $\begin{array}{l}\text { Indian } \\
\text { Chinese } \\
\text { Malay } \\
\text { Chinese } \\
\text { Indian } \\
\text { Chinese } \\
\text { Chinese } \\
\text { Chinese } \\
\text { Caucasian } \\
\text { Indian }\end{array}$ & $\begin{array}{l}\text { Road traffic accident } \\
\text { Road traffic accident } \\
\text { Murder by stabbing } \\
\text { Suicide-jumped from building } \\
\text { Suicide-jumped from building } \\
\text { Suicide-jumped from building } \\
\text { Suicide-jumped from building } \\
\text { Suicide-drowning } \\
\text { Industrial accident } \\
\text { Suicide-hanging }\end{array}$ & $\begin{array}{l}++ \\
+++ \\
+++ \\
+++ \\
++ \\
++ \\
+++ \\
+++ \\
0 \\
0\end{array}$ & $\begin{array}{r}+ \\
0 \\
+ \\
0 \\
0 \\
0 \\
+ \\
++ \\
+ \\
0 \\
0\end{array}$ & $\begin{array}{r}0 \\
0 \\
0 \\
+ \\
+ \\
0 \\
0 \\
0 \\
++ \\
++\end{array}$ & $\begin{array}{r}0 \\
0 \\
0 \\
0 \\
+ \\
0 \\
+++ \\
0 \\
0\end{array}$ & $\begin{array}{r}0 \\
0 \\
0 \\
0 \\
0 \\
0 \\
0 \\
0 \\
++ \\
0\end{array}$ & $\begin{array}{r}0 \\
++ \\
0 \\
0 \\
+ \\
0 \\
0 \\
0 \\
0 \\
0\end{array}$ & $\begin{array}{l}\text { Minimal lesion; FGS* } \\
\text { Minimal lesion } \\
\text { Minimal lesion; MD } \\
\text { Minimal lesion; FGS* } \\
\text { Minor change; MD } \\
\text { Minimal lesion; FGS* } \\
\text { Minimal lesion; MD } \\
\text { DMP; MD; FGS* } \\
\text { Minimal lesion } \\
\text { Minimal lesion; FGS* }\end{array}$ \\
\hline
\end{tabular}

FGS = focal global sclerosis.

$\mathrm{MD}=$ mesangial deposit.

DMP = diffuse mesangial cell proliferation .

"Do not exceed age-related incidence of sclerotic glomeruli in normal population."

where. ${ }^{9}$ All antisera were obtained commercially (Hoechst-Behring Laboratories), and all specimens were stained with rabbit antisera to human IgG, A, M, D, E, C3, C1q, C4, fibrin, albumin, and HBsAg. The intensity and extent of distribution of immunofluorescence was recorded semiquantitatively as negative $(\mathrm{O})$, trace $(\mathrm{O}+)$, mild $(+)$, moderate $(++)$, heavy $(+++)$. To compensate for possible increased background fluorescence of necropsy specimens, sections with trace positive fluorescence were excluded.

\section{Results}

IMMUNOFLUORESCENCE MICROSCOPY

Mesangial IgA as the predominant or sole

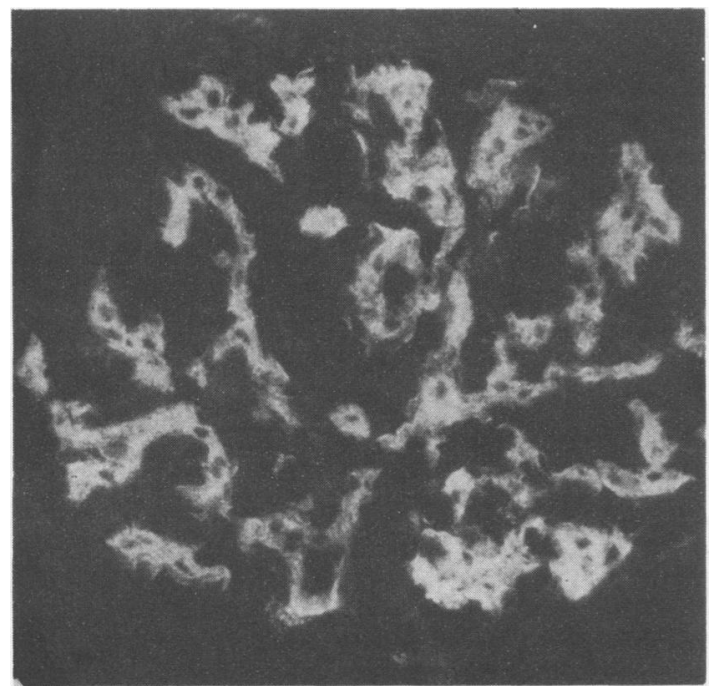

Fig. 1 Heavy (grade +++ ) mesangial IgA deposits in a diffuse mesangial distribution, and in some capillary loops. Immunofluorescence microscopy $\times 250$. immunoglobulin was found in $8(4 \%)$ of the 200 cases, while mesangial IgM and $\mathrm{C1q}$ were seen in two (1\%) cases respectively. Deposits of $\mathrm{C} 3$ in arterioles alone was found in nine $(4.5 \%)$ patients. Details of the patients with predominant mesangial IgA and IgM deposits are summarised in the Table. The deposits of IgA were found as heavy amorphous deposits (Fig. 1) in a diffuse mesangial pattern along all the glomerular lobular stalks, or as granular mesangial deposits. The accompanying IgG, IgM, C3 and fibrin deposits when present were located in the same position, but their

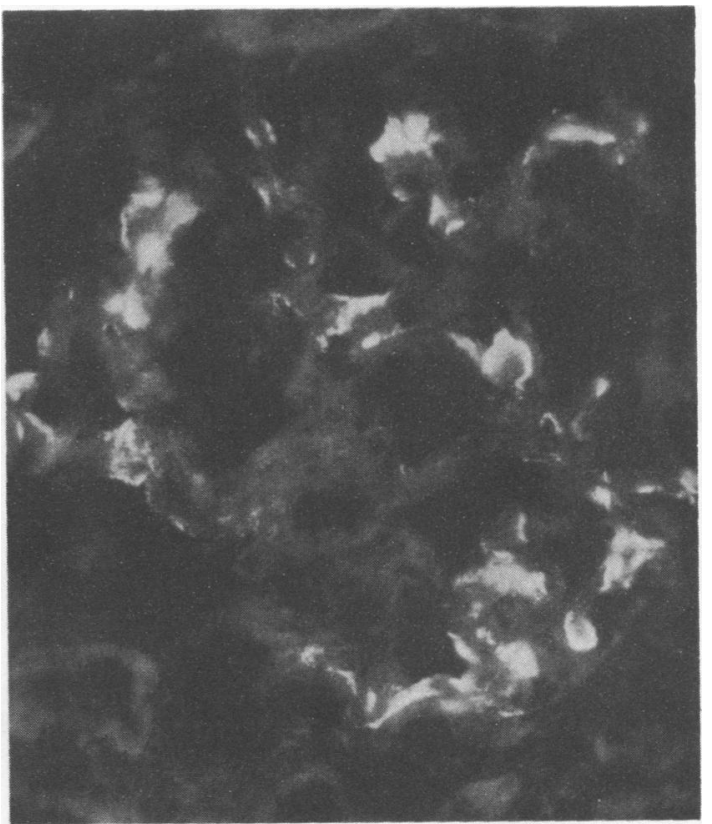

Fig. 2 Moderate (grade ++ ) mesangial IgM deposits in a granular distribution. Immunofluorescence microscopy $\times 250$. 
intensity of fluorescence did not exceed $\operatorname{IgA}$. In the two cases with predominant mesangial IgM deposits, the immunoproteins were located in the mesangium as coarse granular deposits (Fig. 2). C3 was not a constant accompanying feature, and was found in only three of the eight cases with predominant mesangial IgA deposits.

\section{LIGHT MICROSCOPY}

The renal tissues were classified as minimal lesion when the glomeruli were essentially normal with a maximum of two nuclei in the intercapillary space without an increase in mesangial matrix. In minor change, the peripheral mesangial areas contained up to two to three nuclei per mesangial area, and widening of the mesangium up to twice normal.

Details of the glomerular histology in the 10 cases with predominant mesangial IgA and mesangial IgM deposits are summarised in the Table. All the kidney specimens contained 50 to 100 glomeruli in the tissue sections. Of the eight cases with predominant mesangial IgA deposits, six showed minimal lesion, one with minor changes, and one (case 8) showed diffuse mesangial cell proliferation with widening of the centrilobular mesangial stalks (Fig. 3). With the use of Masson's trichrome, PAS and Martius scarlet blue (MSB) stains, mesangial deposits were identified in three cases with minimal or minor lesions, and the one patient with diffuse mesangial cell proliferation. The two subjects with predominant mesangial IgM deposits showed minimal lesions only, and no deposits could be identified. No glomerulus in any specimen showed segmental sclerosis and/or hyalinosis. Total or globally sclerotic glomeruli wereo present in five cases and were randomly distributed and did not exceed the age-related incidence of sclerotic glomeruli in $95 \%$ of the normal popu-o응 lation. ${ }^{11}$ Red cell casts were found in the tubules of $\mathcal{O}$ three of the eight cases with mesangial IgA deposits. $\overrightarrow{0}$ No pigment casts were found in the postmortem 3 kidneys.

\section{Discussion}

Since the first reported series of mesangial $\operatorname{IgA} 3$ nephropathy ${ }^{1}$ there has been considerable interest in this condition. A study conducted in the Singapore 3 Army showed asymptomatic proteinuria oro microscopic haematuria with proteinuria in $2.1 \%$ of all recruits (1410 of 67695 recruits). Mesangial IgA aso the predominant immunoglobulin was found in $56.2 \%$ of 96 cases with haematuria, and in $34.3 \%$ of 35 patients with proteinuria. ${ }^{910}$ It was the commonest 0 form of primary glomerulonephritis accounting forN $33.7 \%$ (239) of all cases in whom renal biopsies were ${ }_{\sigma}^{\omega}$ performed. ${ }^{8}$ The present study shows mesangial IgAdeposits to be a frequent occurrence with a? prevalence of $4 \%$ in an apparently "normal" population. There is no doubt that mesangial $\operatorname{IgA}{ }_{0}^{-}$ deposition shows an unusually high occurrence in Singapore, and the significance of this finding is of great importance in the proper evaluation and $\frac{P}{\mathbb{D}}$ understanding of the renal pathology termed by some as Berger's disease. 
Long term follow-up studies of primary mesangial IgA nephropathy with clinical symptoms have shown that 15 to $20 \%$ progress to chronic renal failure. ${ }^{24}$ With $4 \%$ of our apparently "normal" population having mesangial IgA deposits, renal failure should theoretically be the major cause of death in Singapore. However, disease of the genitourinary tract, were only the ninth major cause of deaths by broad groups of causes in Singapore per 100000 population during the period 1973 to 1978; glomerulonephritis was the major antecedent disease leading to renal death. Glomerulonephritis as a cause of death ranged from $8 \cdot 5$ to $10 \cdot 8$ deaths per 100000 population. ${ }^{12}$ It is reasonable to assume that only a minority of subjects with mesangial IgA deposits develop symptoms, and only a proportion of them eventually die with renal failure.

Predominant mesangial IgM deposits were found in four of 96 patients with asymptomatic microscopic haematuria, and in two of 35 subjects with asymptomatic persistent proteinuria. $^{910}$ In the present control necropsy population study mesangial IgM deposits were found to occur in two of the 200 subjects with no clinical evidence of renal or other organic disease. The frequent occurrence of mesangial $\operatorname{IgA}$ deposits in patients with primary idiopathic glomerulonephritis in France, Japan, Singapore, and in the present study of an apparently normal control necropsy population show without any doubt the high prevalence of these deposits in the local population. There may be some common feature of the antigen(s) or complex involved in the deposition of IgA and $\mathrm{C} 3$ in the local population. This may possibly explain the discrepancy in the findings of IgA glomerular deposits in the local control necropsy population and that reported from the West. ${ }^{13}$ The antigenic stimulation is likely to be of environmental origin, with possibly dietary influences or infective agents in the respiratory and gastrointestinal tracts. Investigations along these lines may help to unravel the enigma of mesangial IgA nephropathy. It is possible that many of these "spontaneous" deposits in the glomerular mesangium result from the clearance of circulating non-nephritogenic immune complexes, mechanisms similar to those occurring in the reticuloendothelial system.
This work was supported by grants from the late PB Davar Memorial Fund, The National University of Singapore and The Ministry of Health, Singapore. I wish to thank Clinical Associate Professor TC Chao, Senior Forensic Pathologist of the Ministry of Health, Singapore for his generous support in allowing access to all the case materials and clinical records; and the technical staff of the University Department of Pathology.

\section{References}

' Berger J, Hinglais N. Les dépôts intercapillaires d'IgA-IgG. J Urol Nephrol (Paris) 1968;74:694-5.

${ }^{2}$ Berger J. IgA glomerular deposits in renal disease. Transplant Proc 1969;1:939-44.

${ }^{3}$ Morel-Maroger L, Leathem A, Richet G. Glomerular abnormalities in non-systemic diseases. Am J Med 1972;53:17084.

4 Droz D. Natural history of primary glomerulonephritis with mesangial deposits of IgA. Contrib Nephrol 1976;2:150-7.

s Sakai O, Yamagata M, Kitajima T, Suzuki T, Ueda Y. Mesangial IgA-IgG deposits nephritis. VI International Congress of Nephrology, Firenze, Italy 1975; Abstract 352, 1975.

- Shirai T, Tomino Y, Sato M, Yoshiki T, Itoh T. IgA nephropathy: clinicopathology and immunopathology. Contrib Nephrol 1978;9:88-100.

7 Yokoska H, Nagase M, Maeda T, Koide K. Mesangial IgA glomerulonephritis: clinicopathological study of 85 cases. Contrib Nephrol 1978;9:101-10.

sinniah R, Javier AR, Ku G. The pathology of mesangial IgA nephritis with clinical correlation. Histopathology 1981;5:469 90.

- Sinniah R, Pwee HS, Lim CH. Glomerular lesions in asymptomatic microscopic hematuria discovered on routine medical examination. Clin Nephrol 1976;5:216-28.

${ }^{10}$ Sinniah R, Law CH, Pwee HS. Glomerular lesions in patients with asymptomatic persistent and orthostatic proteinuria discovered on routine medical examination. Clin Nephrol 1977;7:1-14.

" Kaplan C, Pasternack B, Shah H, Gallo G. Age-related incidence of sclerotic glomeruli in human kidneys. Am $J$ Pathol 1975;00:227-34.

12 Sinniah R. Renal disease in Singapore with particular reference to glomerulonephritis in adults. Singapore Med J 1980;21:583-91.

${ }^{13}$ Larsen S. Glomerular immune deposits in kidneys from patients with no clinical or light microscopic evidence of glomerulonephritis. Acta Pathol Microbiol [A] 1979;87:313-9.

Requests for reprints to: Professor R Sinniah, Department of Pathology, National University of Singapore, Singapore General Hospital, Singapore 0316. 